\title{
Ultra-luminous Supersoft X-ray Sources in Nearby Galaxies
}

\author{
Albert Kong ${ }^{1}$ and Rosanne Di Stefano ${ }^{2}$ \\ ${ }^{1}$ MIT Kavli Institute for Astrophysics and Space Research, 77 Massachusetts Avenue, \\ Cambridge, MA 02139, U.S.A. \\ email: akong@space.mit.edu \\ ${ }^{2}$ Harvard-Smithsonian Center for Astrophysics, 60 Garden Street, Cambridge, \\ MA 02138, U.S.A. \\ email: rd@cfa.harvard.edu
}

\begin{abstract}
Chandra and XMM-Newton improve our understanding of X-ray populations in galaxies. In particular, there exists a class of ultra-luminous X-ray sources (ULXs) for which the observed luminosity is greater than $10^{39} \mathrm{ergs} \mathrm{s}^{-1}$. ULXs are of great interest since they represent a population of possible intermediate-mass black holes. While the spectra of majority of ULXs are similar to Galactic X-ray binaries, a few ULXs have very soft X-ray emission $(k T=50-100 \mathrm{eV})$ resembling supersoft X-ray sources (SSSs) discovered in the Milky Way and the Magellanic Clouds. We here report some recent multiwavelength observations of three ultra-luminous SSSs in M101, NGC 300, and the Antennae. They have shown many interesting behaviors such as state transitions, spectral changes, and time variabilities in different timescales. Unlike typical SSSs, ultra-luminous SSSs are unlikely associated with white dwarfs because of the high X-ray luminosities. We discuss some binary models involving stellar-mass and intermediate-mass black holes to explain the nature of the systems.
\end{abstract}

Keywords. accretion, black hole physics, X-ray: binaries.

\section{Introduction}

Recent high angular resolution X-ray observations reveal that there is a large number of ultra-luminous X-ray sources (ULXs) in many nearby galaxies. ULXs are luminous $\left(L_{X}>10^{39}\right.$ ergs s$\left.^{-1}\right)$ non-nuclear X-ray point sources with apparent X-ray luminosities above the Eddington limit for a $\sim 10 M_{\odot}$ black hole $(\mathrm{BH})$. While some ULXs have been associated with supernovae, many are thought to be accreting objects with X-ray flux variability observed on timescales of hours to years. A natural possibility is that the compact object is an intermediate-mass black hole (IMBH) with mass of $\sim 10^{2-4} M_{\odot}$ (Miller \& Colbert 2004). The origin of such objects remains uncertain. Some ULXs may have a stellar-mass BH with beamed emission (King et al. 2001; Körding et al. 2002). While current observations are inconclusive about the nature of ULXs, recent X-ray observations show that some ULXs have a cool accretion disk $(k T \sim 0.1 \mathrm{keV})$, suggesting the presence of IMBHs (Miller et al. 2003, 2004; Wang et al. 2004).

While the majority of ULXs has X-ray emission from 0.1 to $10 \mathrm{keV}$ and the high energy tail contributes significant emission, a few ULXs have very soft spectra with no X-ray emission above $1 \mathrm{keV}$ (Fabbiano et al. 2003; Kong \& Di Stefano 2003; Di Stefano \& Kong 2003), similar to supersoft X-ray sources (SSSs) in the Milky Way and the Magellanic Clouds. The high luminosities of ultra-luminous SSSs are inconsistent with typical nuclear burning white dwarf models for Galactic SSSs. These ultra-luminous SSSs could, however, very well be IMBHs. Their luminosities and temperatures are consistent with what is predicted for accreting BHs with masses between roughly 100 and $1000 M_{\odot}$. 
Alternatively, outflows from stellar-mass BHs could also achieve such a high luminosity and low temperature (King \& Pounds 2003). We here review 3 well studied ultra-luminous SSSs in nearby galaxies.

\section{M101 ULX-1}

M101 ULX-1 is one of the most luminous ULXs. It was discovered with ROSAT and was confirmed as a SSS with a blackbody temperature of about $100 \mathrm{eV}$, with Chandra (Pence et al. 2001; Mukai et al. 2003; Di Stefano \& Kong 2003). During 2000 March, Chandra detected it at $L_{X} \sim 4 \times 10^{39} \mathrm{ergs} \mathrm{s}^{-1}$, and then in 2000 October, its luminosity was around $10^{39} \mathrm{ergs} \mathrm{s}^{-1}$. In 2004, Chandra conducted a monitoring program for M101. Figure 1 (left) shows the long-term X-ray lightcurve of M101 ULX-1 during 2000-2005. M101 ULX-1 was near the detection limit during 2004 January, March, and May; the $\mathrm{X}$-ray spectra were harder with a power-law shape (see Figure 1 right), and the X-ray luminosity was about $2 \times 10^{37} \mathrm{ergs} \mathrm{s}^{-1}$, a factor of about $10^{2}$ fainter than that during the outbursts in 2000 (Kong et al. 2004). The source was found to be in outburst during the July 5 observation, with an X-ray luminosity of about $7 \times 10^{39} \mathrm{ergs} \mathrm{s}^{-1}$. Data taken on 2004 July 6,7 , and 8 show that the source was in outburst with a peak bolometric luminosity (for assumed isotropic emission) of about $10^{41} \mathrm{ergs} \mathrm{s}^{-1}$ (Kong et al. 2004). In general, the X-ray spectra are best described with an absorbed blackbody model with temperatures of $\sim 50-100 \mathrm{eV}$ (see Figure 1 right). In addition, we found absorption edges at $0.33,0.57,0.66$, and $0.88 \mathrm{keV}$ in two of the high state spectra. These features may signal the presence of highly ionized gas in the vicinity of the accretor (e.g., warm absorber). A DDT XMM-Newton observation was made on 2004 July 23 and the luminosity was about $6 \times 10^{38} \mathrm{ergs} \mathrm{s}^{-1}$. A harder X-ray spectrum with a power-law tail $(k T=53 \mathrm{eV}$ and $\alpha=0.72$ ) was seen up to $7 \mathrm{keV}$. More recently, Chandra observations made in 2004 September and November indicated that the source returned to the low state with a power-law spectrum and a luminosity of $\sim 2 \times 10^{37} \mathrm{ergs} \mathrm{s}^{-1}$. In 2004 December, the source was in outburst again with very soft spectra $(k T=40-150 \mathrm{eV})$ and a peak bolometric luminosity of about $10^{40} \mathrm{ergs} \mathrm{s}^{-1}$. During the rise of the outburst, the spectra were supersoft with blackbody temperatures of 40-70 eV. The peak of the outburst occurred on 2004 December 30. The source showed a cool accretion disk on 2005 January 1. The $\mathrm{X}$-ray spectrum can be fitted with a disk blackbody model with $k T_{D B B}=0.17 \mathrm{keV}$ which is significantly harder than previous supersoft X-ray spectra. The peak luminosity of the 2004 December outburst is about $2 \times 10^{40} \mathrm{ergs} \mathrm{s}^{-1}$ which is very similar to the 2004 July outburst. A DDT XMM-Newton observation made on 2005 January 8 indicated that the source was slightly brighter than 2005 January $1\left(L_{X}=6 \times 10^{39} \mathrm{ergs} \mathrm{s}^{-1}\right)$ and the X-ray spectrum returned to a supersoft state $(k T=56 \mathrm{eV}$; Kong 2005).

Based on the position provided by Chandra, an optical counterpart of M101 ULX-1 was found in archival Hubble Space Telescope (HST) data. The region of M101 ULX-1 was observed with HST using Wide Field Planetary Camera 2 and Advanced Camera for Surveys in 1994, 1995, and 2002. There is a blue object $(V=23.8, B-V=-0.2)$ within the 0.6" Chandra error circle (Kong et al. 2005; Kuntz et al. 2005). The optical counterpart is also detected with ground-based telescopes (Kong et al. 2005). At the distance of M101 $(d=6.7 \mathrm{Mpc})$, the absolute magnitude corresponds to $M_{V}=-5.3$. The magnitudes and colors of the blue star are consistent with an OB star. The source is also clearly located near star forming regions in a spiral arm from GALEX far UV image (Kong et al. 2005).

The IMBH model is the likely model for M101 ULX-1 since stellar-mass black hole has difficulty in explaining the state transitions, temperature changes, and the extremely 

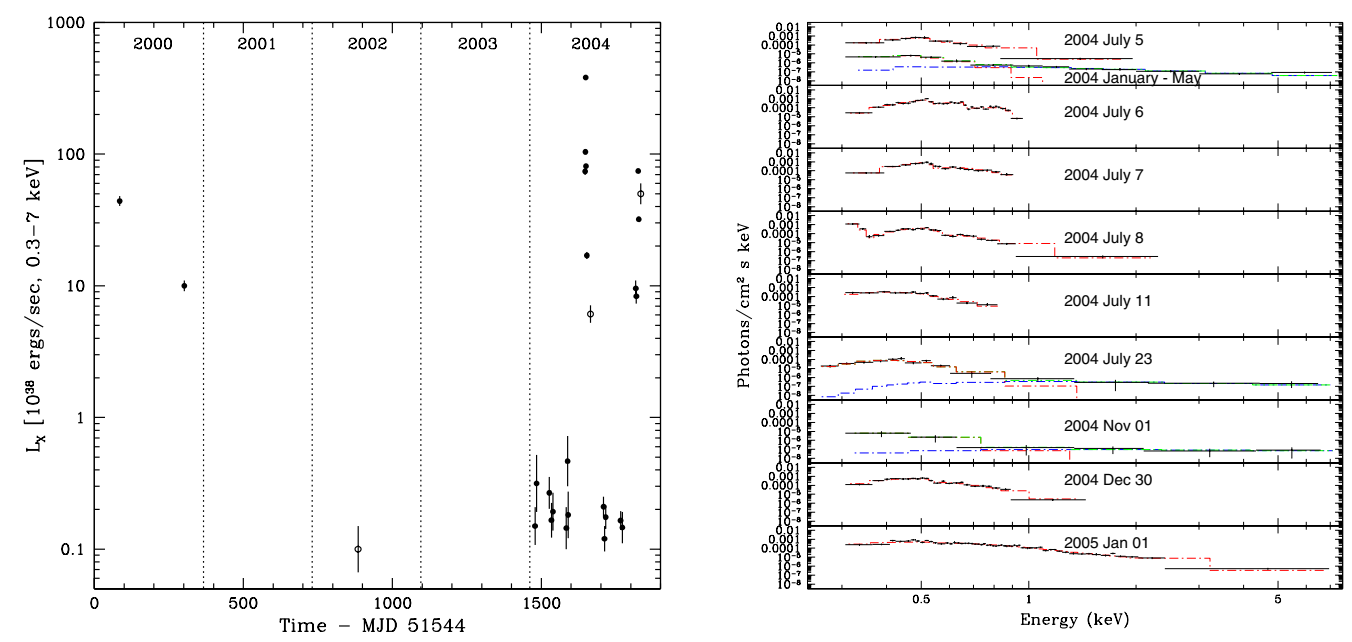

Figure 1. Left: Long-term X-ray light curve of M101 ULX-1 during 2000-2005. The 4 outbursts are clearly shown. The time between outbursts in 2000 was about 210 days, in 2004 this was about 200 days. Right: Unfolded spectra of M101 ULX-1. The total spectrum, blackbody component, and power-law component are shown in green, red, and blue, respectively. During the 2004 July outburst, the spectra can be fitted with blackbody/disk blackbody model with temperatures of $50-100 \mathrm{eV}$. On July 7 , the peak bolometric luminosity reached $10^{41} \mathrm{ergs} \mathrm{s}^{-1}$. The XMM-Newton spectrum taken on July 23 can be fitted with a blackbody $(k T=53 \mathrm{eV})$ and a power-law $(\alpha=0.72)$ model. The combined Jan-May spectrum and the Nov 1 spectrum are similar to the XMM-Newton spectrum, but with much lower luminosities $\left(\sim 10^{37} \mathrm{ergs} \mathrm{s}^{-1}\right)$. The second outburst in 2004 occurred in December. The December 30 spectrum can be fitted with a blackbody model with $k T=70 \mathrm{eV}$. On 2005 January 1, the source is hotter with $k T=150 \mathrm{eV}$.

high luminosities. The location of M101 ULX-1 near star formation regions also favors a high mass companion orbiting around an IMBH.

\section{XMMU J005510.7-373855 in NGC 300}

By using archival XMM-Newton observations, a very luminous SSS ( $k T \approx 60 \mathrm{eV}$ ) was found in NGC 300; the X-ray luminosity is $10^{39} \operatorname{ergs~s}^{-1}$ during the "high" state and $10^{38} \mathrm{ergs} \mathrm{s}^{-1}$ in the "low" state (Kong \& Di Stefano 2003). This SSS is the nearest ultraluminous SSS and is one of the two recurrent ultra-luminous SSSs (Kong et al. 2004). The source was previously seen with ROSAT in 1992 and fell below the detection limit in subsequent ROSAT observations (Fig. 2 left). It reappeared in XMM-Newton observations with bolometric luminosities between $10^{38}$ and $10^{39} \mathrm{ergs} \mathrm{s}^{-1}$. More importantly, the source showed a 5.4-hr periodicity (Fig. 2 right). The high X-ray variability on both short timescales (hours) and long timescales (days to years) indicates that the SSS is an accreting system. If the 5.4-hr variability is the orbital period of the binary system, the $\mathrm{X}$-ray emission can in principle be explained by accretion onto a white dwarf, a neutron star or a BH (see Kong \& Di Stefano 2003 for details). However, the high luminosity in the "high" state makes accreting white dwarf model unlikely, while the supersoft spectra are difficult to be explained by a neutron star accretor. There is no Chandra observation of NGC 300 and it is difficult to locate the optical counterpart in the crowded region by using the XMM-Newton position. A planned Chandra observation in 2006 will help us to resolve this problem and provide important information about the nature of the source. 

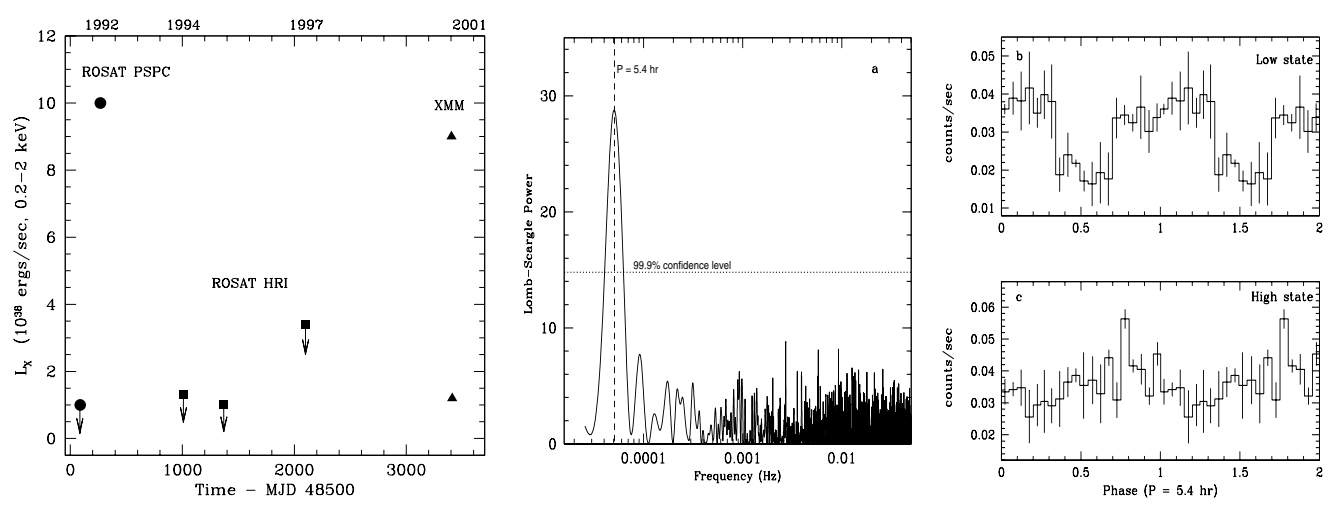

Figure 2. Left: Long-term light curve of the ultra-luminous SSS in NGC 300 from 1991 to 2001 (filled circles: ROSAT PSPC; filled squares: ROSAT HRI; filled triangles: XMM-Newton). Right: The Lomb-Scargle periodogram as obtained by XMM-Newton on 2001 January. The horizontal dotted line is the $99.9 \%$ confidence level. (b) Folded light curve of the low state (2001 January) data on a period of $5.4 \mathrm{hr}$. (c) Folded light curve of the high state (2000 December) data on a period of $5.4 \mathrm{hr}$. The $T_{0}$ of both light curves are set at the time of the first data point.

\section{CXOAnt J120151.6-185231.9 in the Antennae}

This SSS is one of the earliest ultra-luminous SSSs discovered by Chandra (Zezas et al. 2002). During a monitoring program between 1999 and 2002, the source was detected in all four Chandra observations. The luminosity varied between $10^{38} \mathrm{ergs} \mathrm{s}^{-1}$ and $10^{40} \mathrm{ergs} \mathrm{s}^{-1}$ and the temperature was between $90 \mathrm{eV}$ and $150 \mathrm{eV}$ (Fabbiano et al. 2003). There was no obvious optical counterpart from HST at the position of the SSS (Zezas et al. 2002). While IMBH model is a natural explanation if we assume an isotropic emission, stellar-mass BH model is feasible if the emission is mildly anisotropic resulting from accretion at close to the Eddington rate onto the $\mathrm{BH}$. White dwarf is also a possible solution but it requires extreme beaming.

\section{References}

Di Stefano, R. \& Kong, A. K. H. 2003, ApJ, 592, 884

Fabbiano, G., et al. 2003, ApJ, 591, 843

Kong, A. K. H. \& Di Stefano, R. 2003, ApJ, 590, L13

Kong, A. K. H., Di Stefano, R., \& Yuan, F. 2004, ApJ, 617, L49

Kong, A. K. H. 2005, ATel, 409

Kong, A. K. H., Rupen, M. P., Sjouwerman, L. O., \& Di Stefano, R. 2005, in the proceedings of the XXII Texas Symposium on Relativistic Astrophysics, Stanford University (astro$\mathrm{ph} / 0503465)$

Körding, E., Falcke, H., \& Markoff, S. 2002, A\&A, 382, L13

King, A. R., Davies, M. B., Ward, M. J., Fabbiano, G., \& Elvis, M. 2001, ApJ, 552, L109

King, A. R. \& Pounds, K. A. 2003, MNRAS, 345, 657

Kuntz, K. D., et al. 2005, ApJ, 620, L31

Miller, M. C. \& Colbert, E. J. M. 2004, International Journal of Modern Physics D, 13, 1

Miller, J. M., Fabbiano, G., Miller, M. C., \& Fabian, A. C 2003, ApJ, 585, L37

Miller, J. M., Fabian, A. C., \& Miller, M. C. 2004, ApJ, 614, L117

Mukai, K., Pence, W. D., Snowden, S. L., \& Kuntz, K. D. 2003, ApJ, 582, 184

Pence, W. D., Snowden, S. L., Mukai, K., \& Kuntz, K. D. 2001, ApJ, 561, 189

Wang, Q. D., Yao, Y., Fukui, W., Zhang, S. N., \& Williams, R. 2004, ApJ, 609, 113

Zezas, A., Fabbiano, G., Rots, A. H., \& Murray, S. 2002, ApJS, 142, 239 


\section{Discussion}

BARNARD: We have observed several supersoft sources with back body temperatures of $50 \mathrm{eV}$ and apparent luminosities of $10^{40}-10^{41} \mathrm{erg} / \mathrm{s}$. However, with other thermal models, e.g. NSA model, the luminosity can be 5-6 orders of magnitude lower.

KonG: I totally agree that we have to examine every possible spectral model to derive the best fit and the correct luminosity.

MiraBeL: Could SS433 be an Ultraluminous Supersoft source?

KonG: If SS433 satisfies the condition of ultraluminous supersoft X-ray source (i.e. the spectrum can be fit with a thermal model with $\mathrm{kT}<0.1 \mathrm{keV}$ and has a luminosity of $>10^{39} \mathrm{erg} / \mathrm{s}$ ), then SS433 can be an ultraluminous SSS. 